\title{
Relationship between meeting the recommendations for physical activity and health-related quality of life in adult Chinese Internet users
}

\author{
Jia-Meng $\mathrm{Ma}^{1,2^{\star}}$, Ai Shibata ${ }^{3}$, Isao Muraoka ${ }^{3}$ \\ ${ }^{1}$ Graduate School of Sport Sciences, Waseda University, Tokyo, Japan; *Corresponding Author: jiameng2011@gmail.com \\ ${ }^{2}$ School of Physical Education, Sendai University, Sendai, Japan; \\ ${ }^{3}$ Faculty of Sport Sciences, Waseda University, Tokyo, Japan; aishibata@aoni.waseda.jp, imuraoko@aoni.waseda.jp
}

Received 10 September 2011; revised 17 October 2011; accepted 29 October 2011.

\section{ABSTRACT}

Background: The benefits of a recommended level of physical activity on physiological health indicators such as morbidity and mortality are well-accepted, but few researches has addressed whether or not the association between the recommended level of physical activity and a health-related quality of life (HRQOL) exists in the Chinese adults. Purpose: The present study examined whether the recommended physical activity (PA) would be associated with HRQOL in the Chinese adults. Methods: Cross-sectional data were collected through an internet-based survey. Total of 1394 Chinese adults responded the International Physical Activity Questionnaire-Chinese version examining whether individuals met the recommended ACSMIAHA PA guideline. Demographic data were also obtained. HRQOL was assessed with the Medical Outcomes Survey Short Form-36 questionnaire (SF36). Multivariate analyses of covariance were utilized to examine differences in multidimensional scales of the SF-36. Results: In both genders, the recommended group had significantly higher physical functioning, vitality, and mental health scores than the inactive group. Significant differences in role physical, general health, social functioning scores were only found among the recommended and insufficient male groups. Conclusion: Individuals who attained the recommended level of PA had better scores on some dimensions of HRQOL than those who did not.

Keywords: Behavior Science; Health Promotion; HRQOL; Physical Activity

\section{INTRODUCTION}

In its focus on healthy living, the World Health Organization (WHO) places an emphasis not simply on preventing chronic diseases, but on preserving or improving quality of life (QOL) through the maintenance of a good state of physical independent function and mental health in daily life. Health-related quality of life (HRQOL) is defined as individual and community perceptions of physical and mental health, and is considered an important indicator in evaluating health status [1]. It is also a basic tool used to evaluate self-awareness of the physical and mental aspects of health status in daily life $[2,3]$.

Sufficient physical activity is already known to reduce the risk of mortality and morbidity of chronic conditions such as coronary artery disease, diabetes mellitus, or hypertension [4-7]. It is also well-documented that as well as having such physical effects, physical activity also has mental effects. Previous studies have reported that people who are physically inactive in their daily lives have lower HRQOL than active people [8-10], and that the mental aspect of HRQOL in those who engage in regular exercise is superior to that of those with a sedentary lifestyle [11]. Based on these findings, health care providers and health promotion workers recommend engaging in regular physical activity as a means to maintain or improve HRQOL.

In the United States (US), the American College of Sports Medicine/American Heart Association (ACSM/ AHA) published guidelines encouraging people to participation physical activity in order to prevent chronic diseases and maintain or improve health [12]. These guidelines recommend engaging in $\geq 150$ minutes per week of at least physical activity of moderate-intensity, and the guidelines are currently in widespread use around the world $[13,14]$. In a study using the Medical Outcomes Study short form 36 (SF-36) to investigate the 
relationship between meeting the recommended amount of physical activity and HRQOL in French adults, those who met the recommended amount scored higher than those who did not in five of the eight SF-36 domain scales: physical functioning, vitality, general health, social functioning, and mental health $[12,15]$. Thus, reports mainly from the US and Europe indicate that meeting the recommended amount of physical activity not only reduces the prevalence of chronic diseases or the mortality rate from such diseases, but is also beneficial in increasing the HRQOL[10].

China also has the same emphasis on HRQOL as other countries. In the Healthy China 2020 Strategy announced by the Ministry of Health of the People's Republic of China, the ultimate goal of health promotion is to increase HRQOL [16]. While there have been occasional studies in China of the relationship between HRQOL and physical activity focusing on specific groups such as people suffering a particular disease [17,18], there have been no studies investigating this relationship in Chinese adults across a wide range of age-groups [19].

There has been tremendous socioeconomic growth in China in recent, particularly in urban areas, and lifestyles are becoming westernized at an ever-increasing rate. According to a 2009 survey report of the development of the Internet in China, the number of Internet users in urban areas is increasing every year. Internet penetration in China is $31.8 \%$, and $72.6 \%$ of Internet users are urban dwellers. Health problems among these urban Internet users are expected to increase as a result of increasing Westernization of their lifestyles and decreased physical activity. In order to build the measures to address physiccal inactivity, it would be useful to survey physiccal activity and HRQOL among urban dwellers in China, who are at particularly high risk of physical inactivity.

The present study examined that the relationship between recommended amount of physical activity and HRQOL among adult Chinese Internet users.

\section{METHODS}

\subsection{Participants}

The survey was carried out from December 2009 to January 2010. The participants were Chinese adults registered as monitors (approx. 1,950,000 people as of November, 2009) with a Japanese company specializing in overseas surveys. The goal was to have responses from 1,300 people, and after stratifying the participants to ensure equal numbers of each gender and age group (30 - 39 years, 40 - 49 years, 50 - 59 years), 39,000 adults from across China were randomly selected. The survey company sent an e-mail to each person requesting their cooperation with the questionnaire survey. The final number of respondents was 1501 , a response rate of $3.8 \%$. By way of remuneration, the participants who responded to the questionnaire survey were awarded Internet points by the survey company according to the number of survey items to which they responded. The present study was approved by the institutional review board by the Waseda University (approved number 2009-145, Dec. 7. 2009).

\subsection{Measures}

\subsubsection{Physical Activity}

The Chinese version of the International Physical Activity Questionnaire-Short Version (IPAQ-SV) [20] was used to estimate the amount of physical activity. The IPAQ-SV was designed for the purpose of identifying the frequency and duration of walking, moderate and vigorous physical activity, and sedentary activity during the past week The reliability and validity of the Chinese version of the IPAQ-SV have already been investigated in a previous study, with a test-retest reliability coefficient of $r=0.779$ and validity shown by a good correlation of $r=0.598$ with the daily energy expenditure calculated from the physical activity log [20]. This is a similar result to verification of the reliability and validity of the International Physical Activity Questionnaire carried out in 12 countries [21].

The total amount of physical activity (hours/week) was weekly minutes of walking, moderate-intensity and vigorous-intensity activity were calculated by multiplying the number of days/week by the frequency of physiccal activity. On the basis of the ACSM/AHA guidelines, participants with a total weekly amount of physical activity (including walking and moderate-intensity and vigorous intensity physical activity) of 150 minutes or more were classified into the sufficient group, and those with a total of less than $150 \mathrm{~min}$ were classified into the insufficient group.

\subsubsection{HRQOL}

The Chinese version of the SF-36 [22] was used to measure HRQOL. The validity and reliability of applying this instrument to Chinese people have been confirmed [23]. It has also been shown that the electronic version, used over media such as the Internet, has the same levels of validity and reliability as the paper selfreporting version [24]. The questionnaire has 36 items, organized into the following eight domain scales: physiccal functioning $(\mathrm{PF})$, role-physical (RP), bodily pain (BP), general health $(\mathrm{GH})$, vitality (VT), social functioning (SF), role-emotional (RE), and mental health $(\mathrm{MH})$. Each domain scale is calculated as a score from 0 
to 100 , with higher scores indicating better levels of subjective health and functioning.

\subsection{Statistical Analysis}

Of the 1501 respondents, 107 were excluded on the basis of the data exclusion criteria established in the IPAQ-SV protocol, including cases in which the total physical activity exceeded 960 minutes (16 hours) and cases for which data were lost. The remaining 1,394 subjects $(92.9 \%)$ were analyzed. All analyses were performed after stratification by gender. A t-test and a one-way analysis of variance (ANOVA) were used to investigate the relationships between demographic variables and differences in the SF-36 domain scales. The differences in scale scores between the two groups classified according to whether they met the recommended amount of physical activity (sufficient group, insufficient group) were investigated using a multivariate analysis of covariance (MANCOVA), with the demographic variables for which a significant relationship with the domain scales was found in the ANOVA (age, marital status, employment status) as covariates. The significance level was set at $\mathrm{p}<0.05$. The analysis soft- ware was SPSS ver. 17.0

\section{RESULTS}

\subsection{Basic Characteristics of Respondents}

The mean age of subjects was $43.0 \pm 8.0$ years, and $50.2 \%$ were male. The participants were $91.7 \%$ married, $97.8 \%$ employed, and $67.6 \%$ had university or higher educational status. In addition, $62.3 \%$ had a personal annual household income of 50,000 yuan or more and $63.7 \%$ had a body mass index within the standard range. As for walking, total minutes were $280.2 \pm 319.2 \mathrm{~min}$ per week in male, while females accumulated totally $294.8 \pm 373.2$ min per week. Also, males spent $250.6 \pm$ $348.5 \mathrm{~min}$ in moderate-intensity activity, whereas females spent $254.8 \pm 346.6$ minutes. In addition, weekly time of vigorous-intensity activity in males and females were $180.7 \pm 279.0$ minutes and $160.9 \pm 281.6$ minutes respecttively. Of the total attributes, $87.3 \%$ met the recommended amount of physical activity (Table 1). Of these, $89.0 \%$ of males and $85.6 \%$ of females met the recommended amount of physical activity. Moreover, there were $2.6 \%$ of participants were found to be inactive.

Table 1. Basic characteristics and physical activity level for male and female Chinese respondents.

\begin{tabular}{|c|c|c|c|c|c|}
\hline & \multicolumn{4}{|c|}{ Participants } & \multirow{3}{*}{ Total n (\%) } \\
\hline & \multicolumn{2}{|c|}{ Men } & \multicolumn{2}{|c|}{ Women } & \\
\hline & n (\%) & mean(SD) & n (\%) & mean (SD) & \\
\hline Total & $700(50.2)$ & & $694(49.8)$ & & $1394(100)$ \\
\hline \multicolumn{6}{|l|}{ Age } \\
\hline $30-39$ & $234(33.4)$ & & $235(33.9)$ & & $469(33.6)$ \\
\hline $40-49$ & $234(33.4)$ & & $232(33.4)$ & & $466(33.4)$ \\
\hline $50-59$ & $232(33.1)$ & & $227(32.7)$ & & 459 (32.9) \\
\hline married & $650(92.9)$ & & $628(90.5)$ & & $1278(91.7)$ \\
\hline unmarried & $50(7.1)$ & & $66(9.5)$ & & $116(8.3)$ \\
\hline \multicolumn{6}{|l|}{ Employment status } \\
\hline employed & $688(98.3)$ & & $701(97.5)$ & & $1364(97.8$ \\
\hline not employed & $12(1.7)$ & & $18(2.5)$ & & $30(2.2)$ \\
\hline \multicolumn{6}{|l|}{ Educational status } \\
\hline$\leq$ high school graduate & $4(7.0)$ & & $53(7.6)$ & & $102(7.3)$ \\
\hline$\geq$ college graduate & $491(70.0)$ & & $452(65.1)$ & & $943(67.6)$ \\
\hline \multicolumn{6}{|l|}{ Income level (RMB) } \\
\hline$<30,000$ & $90(12.9)$ & & $78(11.2)$ & & $168(12.1)$ \\
\hline $30,000-40,000$ & $75(10.7)$ & & $69(9.9)$ & & $144(10.3)$ \\
\hline$>40,000-50,000$ & $105(15.0)$ & & $108(15.6)$ & & $213(15.3)$ \\
\hline$>50,000$ & $430(61.4)$ & & $439(63.0)$ & & $869(62.3)$ \\
\hline \multicolumn{6}{|l|}{ BMI $\left(\mathrm{kg} / \mathrm{m}^{2}\right)$} \\
\hline$<18.5$ & $10(1.4)$ & & $49(7.1)$ & & $59(4.2)$ \\
\hline $18.5-23.9$ & $398(56.9)$ & & $490(70.7)$ & & $888(63.7)$ \\
\hline $24.0-27.9$ & $252(36.0)$ & & $136(19.6)$ & & $388(27.8)$ \\
\hline$\geq 28.0$ & $40(5.7)$ & & $19(2.7)$ & & $59(4.2)$ \\
\hline \multicolumn{6}{|l|}{ Physical activity level } \\
\hline Recommended $^{\mathrm{a}}$ & $623(89.0)$ & & $594(85.6)$ & & \\
\hline Walking $(\mathrm{min} / \text { week })^{\mathrm{b}}$ & & $280.2(319.2)$ & & $294.8(373.2)$ & \\
\hline Moderate-intensity activity $(\mathrm{min} / \text { week })^{b}$ & & $250.6(348.5)$ & & $254.8(346.6)$ & \\
\hline Vigorous-intensity activity $\left(\mathrm{min} /\right.$ week) ${ }^{b}$ & & $180.7(279.0)$ & & $160.9(281.6)$ & \\
\hline
\end{tabular}

$\mathrm{RMB}=$ China yuan. a. Met the recommended amount of physical activity. $\mathrm{b}$. Mean (SD). 


\subsection{Effects of Physical Activity Level on HRQOL}

Regarding the 8-domain scales scores, a one-way ANOVA was conducted to examine the group differences in the SF-36 measures for each demographic variable. In both genders the differences of PF, BP, and GH with meeting the required amount were lowest in the 50 - 59 years age group and highest in the 30 - 39 years age group. For marital status, unmarried males only showed higher RP than married males. For employment status, $\mathrm{PF}, \mathrm{RP}, \mathrm{GH}, \mathrm{VT}$, and $\mathrm{MH}$ were higher among employed males than unemployed males, and BP and GH were higher among employed females than unemployed females. Also, a MANCOVA was performed with these influential variables (age, marital status, and employment status) as covariants, the scores on the eight domain scales as dependent variables, and the physical activity groups as independent variables. Multivariate effects for PF, RP, GH, VT, SF, and MH were found among males and for PF, VT, and MH among females (males; Wilk's $\lambda=0.934, \mathrm{p}=0.000$; females: Wilk's $\lambda=$ $0.934, \mathrm{p}=0.000$ ). Moreover, a univariate analysis showed that $\mathrm{PF}, \mathrm{VT}$, and $\mathrm{MH}$ were significantly higher in the sufficient group than the insufficient group for both genders. In the insufficient group, RP, GH, and SF were significantly higher in both genders (Table 2).

\section{DISCUSSION}

The present study investigated the relationship between meeting the recommended amount of physical activity and HRQOL among Chinese adults that use the Internet. Overall, $87.3 \%$ of participants met the recommended amount of physical activity. A previous study of physical activity among Chinese adults using the IPAQ-SV found that $88.8 \%$ of adults carried out $\geq 150$ minutes of physical activity per week [25], so the results of the present study were consistent with this previous study. Moreover, with regard to HRQOL, the overall results of the present study were slightly lower than a previous survey of Chinese adults using the SF-36 [26], but the trends in scores for each scale were similar.

Meeting the recommended amount of physical activity had a significant relationship with better scores for almost all domain scales (PR, RP, GH, VT, SF, and MH) among males, even after adjustment for demographic factors. Among females, meeting the recommended amount was significantly related to with better scores for $\mathrm{PF}, \mathrm{VT}$, and $\mathrm{MH}$. The findings of the present study thus imply that engaging in the recommended amount of physical activity is associated with both physical and mental aspects of HRQOL. The SUVIMAX epidemiological cohort study in France also reported that partici-
Table 2. Aadjusted HRQOL measures in respondents among physical activity groups stratified by gender.

\begin{tabular}{|c|c|c|c|c|}
\hline \multicolumn{5}{|c|}{ Physical Activity Group } \\
\hline Male mean (SD) & Recommended & Insufficient & $\mathbf{F}$ & $\mathbf{P}$ \\
\hline $\mathrm{PF}$ & 88.44 (14.6) & $80.71(20.3)$ & 16.45 & $<0.001$ \\
\hline RP & 84.70 (19.6) & $78.99(23.2)$ & 5.70 & 0.017 \\
\hline BP & $77.90(16.8)$ & $76.86(15.0)$ & 0.18 & 0.676 \\
\hline $\mathrm{GH}$ & $67.71(19.7)$ & $56.40(22.8)$ & 19.82 & $<0.001$ \\
\hline VT & $68.64(16.0)$ & $58.79(18.3)$ & 22.29 & $<0.001$ \\
\hline SF & $77.12(17.6)$ & $70.94(18.5)$ & 8.05 & 0.005 \\
\hline $\mathrm{RE}$ & $79.95(20.3)$ & $75.76(22.2)$ & 2.72 & 0.100 \\
\hline MH & $67.17(15.3)$ & $62.14(18.4)$ & 5.41 & 0.020 \\
\hline \multicolumn{5}{|l|}{ Female mean (SD) } \\
\hline $\mathrm{PF}$ & $88.36(12.4)$ & $83.30(17.0)$ & 13.75 & $<0.001$ \\
\hline $\mathrm{RP}$ & $85.98(18.1)$ & $86.32(19.8)$ & 0.01 & 0.934 \\
\hline BP & 77.55 (16.7) & $80.28(18.0)$ & 1.96 & 0.163 \\
\hline $\mathrm{GH}$ & $67.46(20.2)$ & $64.22(19.9)$ & 2.69 & 0.102 \\
\hline VT & $69.08(15.2)$ & $64.40(17.9)$ & 8.33 & 0.004 \\
\hline SF & $78.72(17.1)$ & $81.50(17.0)$ & 1.97 & 0.161 \\
\hline RE & $81.47(18.5)$ & $81.67(21.8)$ & 0.00 & 0.951 \\
\hline MH & $67.59(14.8)$ & $63.05(18.1)$ & 7.11 & 0.008 \\
\hline
\end{tabular}

Comparison in multidimensional scales of SF-36 among physical activity levels withcovariate of age, marital status, employment status. Bonferroniadjusted univariate multiple comparison.

pants engaging in the recommended amount of physical activity showed greater values for PF, RP, GH, RE, VT, $\mathrm{SF}$, and MH than the control group [15]. Furthermore, the Behavioral Risk Factor Surveillance System (BRFSS), a large-scale survey of physical activity conducted in the US, reported that a significantly lower proportion of people who subjectively evaluated themselves as unhealthy for 14 or more days in a month met the recommended amount of physical activity than other people [27]. It therefore appears that meeting the recommended amount of physical activity is associated with better HRQOL among adult Internet users in China in the same way as in developed countries.

Of the eight domain scales, no significant relationship was found between BP or RE and physical activity in males, or between RP, BP, GH, SF, or RE and physical activity in females. In a previous study, vigorous-intensity physical activity is associated with all domains of the SF-36 scale, and that vigorous-intensity activity has a greater effect on all domains in females than in males 
[28]. Another previous study also found that vigorous-intensity leisure-time physical activity was more strongly associated with all of the domains than moderate-intensity physical activity [15]. With the present study, it is not possible to make direct mention to any amount-response relationship between the intensity or amount of physical activity and HRQOL, as the survey examined the relationship between HRQOL and engageing in $\geq 150$ minutes per week of at least physical activity of moderate-intensity. Nonetheless, in light of the previous studies, the present results suggest the possibility that higher intensity or greater amount of physical activeity are required in order to have an effect on the domains in which no relationship with meeting the recommended amount was found, and that carrying out the recommended amount of physical activity of 150 minutes per week of physical activity of moderate-intensity or vigorous-intensity may not be sufficient.

Furthermore, while a significant relationship between physical activity and the SF domain was found among males, no relationship was found among females. In a previous study, a longitudinal study found that five years of continual leisure-time activity had a favorable effect in raising SF in females [29]. The present study was cross-sectional, and, moreover, physical activity habits and period of duration were not surveyed, but it seems that engaging in a constant amount and duration of physical activity has an effect on SF in females. There is therefore a need to investigate this point using a longitudinal study.

Moreover, in the present study, female found a weaker relationship between the physical aspects of HRQOL in particular and meeting the recommended amount of physical activity. A previous study that examined the relationship between long-term physical activity and HRQOL reported that the more intense the physical activity, the stronger the relationship between physical activity and the physical aspects of HRQOL [30]. This suggests that engaging in high-intensity activity has an effect on the physical aspects of HRQOL. There is also a trend for males to favor high-intensity activity, while females generally engaging in moderate-intensity physiccal activity [31]. Consequently, in the present study, it is likely that, among those engaging in $150 \mathrm{~min}$ or more physical activity per week, the males were engaging in high-intensity activity, while more females were engageing in moderate-intensity activity. It would probably be desirable to recommend that female engaging in highintensity activity in order to achieve more effective improvement of the physical aspects.

In a previous study, engaging in leisure-time physical activity of moderate or greater intensity for $150 \mathrm{~min}$ or more per week showed a strong relationship to the
HRQOL domain scales (except BP) [15]. In the present study, however, evaluation of the relationship to HRQOL of physical activity of moderate or greater intensity that includes activities of daily life observed several domain scales for which no relationship was found. From this, it may be conjectured that leisure-time physiccal activity has a greater relation to HRQOL than the general activity of daily life. Moreover, as leisure-time physical activity has a positive effect on overall health, while physical activity from commuting or work has a negative effect on subjective well-being, it has been suggested that physical activity engaging in a passive condition has a great effect on psychological factors [32]. In China, one-third of all people commuting to work go by bicycle or on foot. One would therefore expect that the participants of the present study included many people met physical activity recommendation by such active commuting. Therefore, the finding of the present study that there was no relationship between physical activity and the psychological domains RE and SF was probably due at least in part to differences in the type of exercise being engaging in by participants.

There are a number of limitations to the present study. First, the cross-sectional design of the study makes it impossible to mention a causal relationship between engaging in the recommended amount of physical activeity and HRQOL. Second, there is the presence of sampling bias. Subjects were selected after stratifying by gender and age group, but it has been pointed out that sampling error can occur when subjects are selected by random sampling from monitors registered with a survey company [33]. Comparing the characteristics of the subjects of the present survey with the adult population of China as whole using information released by the $\mathrm{Na}$ tional Bureau of Statistics of China in 2008, the survey participants were better educated and had higher income. Adult Chinese people who use the Internet are those with higher socioeconomic status, so there is probably a bias toward people with the same attributes in the monitors of the survey company. According to a survey by the China Internet Network Information Center (CNNIC), at least $70 \%$ of Internet users are urban dwellers, so that the subjects of the present study were probably a largely urban group. These points mean that caution must be exercised in any attempt to generalize the results of the present survey to the adult population of China as a whole. Furthermore, with regard to the amounts of physical activity of moderate or greater intensity and of walking assumed in the IPAQ, it is impossible to avoid bias due to inaccurate assumptions or recollections on the part of the subjects because of the self-reporting nature of the study. In a previous study of physical activity evaluation methods with Chinese subjects [34], the IPAQ 
gave a higher evaluation of physical activity amount than a heart rate monitor, an accelerometer, and a questionnaire relating to a daily physical activity log. Therefore, the IPAQ may overestimate the amount of physical activity.

\section{CONCLUSIONS}

Since a relationship was found between meeting the amount of physical activity recommended in the ACSM/ AHA guidelines and HRQOL, it is likely that engaging in the recommended amount of physical activity has an important effect on health. However, as discussed above, further studies should be done to determine more accurately the association between HRQOL and the recommended physical activity level using a representative sample and a larger sample size. Participants should not be only restricted to the internet user sample. HRQOL may possibly be related to other characteristics of the Chinese population. Moreover, the association between HRQOL with specific domains of physical activity (such as leisure-time household, occupational and transport) should be examined further. Furthermore, effective support strategies are needed for promoting physical activity with the goal of improving HRQOL.

\section{ACKNOWLEDGEMENTS}

This work was supported by a Global COE program "Sport Sciences for the Promotion of Active Life" from the Japan Ministry of Education, Culture, Sports, Science, and Technology.

\section{REFERENCES}

[1] U.S. Department of Health and Human Services (2000) Measuring healthy days, population assessment of Healthrelated Quality of Life.

[2] Mishoe, S.C. and Maclean, J.R. (2001) Assessment of health-related quality of life. Respiratory Care, 46, 12361257.

[3] Ware, J.E. Jr. and Sherbourne, C.D. (1992) The MOS 36item short-form health survey (SF-36) I. Conceptual framework and item selection. Medical Care, 30, 473-483. doi:10.1097/00005650-199206000-00002

[4] Sundquist, K., Qvist, J., Johansson, S.E. and Sundquist, J. (2005) The long-term effect of physical activity on incidence of coronary heart disease: A 12-year follow-up study. Preventive Medicine, 41, 219-225. doi:10.1016/j.ypmed.2004.09.043

[5] Warburton, D.E.R., Nicol, C.W. and Bredin S.S.D. (2006) Health benefits of physical activity: The evidence. Canadian Medical Association journal, 174, 801-809. doi:10.1503/cmaj.051351

[6] Manson, J.E., Hu, F.B., Rich-Edwards, J.W., Colditz, G.A., Stampfer, M.J. and Willett, W.C. (1999) A prospective study of walking as compared with vigorous exercise in the prevention of coronary heart disease in women.
New England Journal of Medicine. 341, 650-658. doi:10.1056/NEJM199908263410904

[7] Bjorntorp, P. (1992) Abdominal Obesity and the Metabolic Syndrome. Annals of Medicine, 24, 465-468. doi:10.3109/07853899209166997

[8] Tentori, F., Elder, S.J., Thumma, J., Pisoni, R.L., Bommer, J., Fissell, R.B., et al. (2010) Physical exercise among participants in the Dialysis Outcomes and Practice Patterns Study (DOPPS): Correlates and associated outcomes. Nephrology Dialysis Transplantation, 25, 30503062. doi:10.1093/ndt/gfq138

[9] U. S. Department of Health and Human Services (1996) Centers for Disease Control and Prevention, National Center for Chronic Disease Prevention and Health Promotion.

[10] Harrison, R. A., McElduff, P. and Edwards, R. (2006) Planning to win: Health and lifestyles associated with physical activity amongst 15,423 adults. Public Health, 120, 206-212. doi:10.1016/j.puhe.2005.08.021

[11] Lee, C. and Russell, A. (2003) Effects of physical activity on emotional well-being among older Australian women cross-sectional and longitudinal analyses. Journal of Psychosomatic Research, 54, 155-160. doi:10.1016/S0022-3999(02)00414-2

[12] Haskell, W. L., Lee, I. M., Pate, R. R., Powell, K. E., Blair, S. N., Franklin, B. A., et al. (2007) Updated recommendation for adults from the American College of Sports Medicine and the American Heart Association. Circulation, 116, 1081-1093. doi:10.1161/CIRCULATIONAHA.107.185649

[13] Meseguer, C.M., Galán, I., Herruzo, R., Zorrilla, B. and Rodríguez-Artalejo, F. (2009) Leisure-time physical activity in a southern European mediterranean country: adherence to recommendations and determining factors. Revista espanola de cardiologia, 62, 1125-1133. doi:10.1016/S0300-8932(09)72381-4

[14] Stevinson, C., Capstick, V., Schepansky, A., Tonkin, K., Vallance, J.K., Ladha, A.B. et al. (2009) Physical activity preferences of ovarian cancer survivors. Psychooncology, 18, 422-428. doi:10.1002/pon.1396

[15] Vuillemin, A., Boini, S., Bertrais, S., Tessier, S., Oppert, J.M., Hercberg, S., et al. (2005) Leisure time physical activity and health-related quality of life. Preventive Medicine, 41, 562-569. doi:10.1016/j.ypmed.2005.01.006

[16] Huang, Y.X., Zeng, Y.X. and Kuang, L. (2008) To estimate the engaging in effect and policy choice of the health care financing blue print on "Health China by the Year 2020". Chinese Health Economics, 5, 9-12.

[17] Qian, Y., Zhang, J., Lin, Y., Dong, M., Xu, M., Wu, L., et al. (2009) A tailored target intervention on influence factors of quality of life in Chinese patients with hypertension. Clinical and Experimental Hypertension, 31, 71-82. doi:10.1080/10641960802409804

[18] Chan, C., Tang, D. and Jones, A. (2008) Clinical outcomes of a Cardiac Rehabilitation and Maintenance Program for Chinese patients with congestive heart failure. Disability and rehabilitation, 30, 1245-1253. doi:10.1080/09638280701580226

[19] Schooling, C.M., Lam, T.H., Li, Z.B., Ho, S.Y., Chan, W.M, Ho, K.S., et al.(2006) Obesity, physical activity, and mortality in a prospective Chinese elderly cohort. Archives of Internal Medicine, 166, 1498-1504. 
doi:10.1001/archinte.166.14.1498

[20] Qu, N.G. and Li, K.J. (2004) Study on the reliability and validity of international physical activity questionnaire (Chinese version IPAQ).Chinese Journal of Epidemiology, 25, 265-268.

[21] Craig, C.L., Marshall, A.L., Sjöström, M., Bauman, A.E., Booth, M.L., Ainsworth, B.E., et al. (2003) International physical activity questionnaire. 12-country reliability and validity. Medical Science Sports Exercise, 35, 1381-1395.

[22] Li., L., Wang. H. and Shen, Y. (2003) Chinese SF-36 Health Survey: Translation, cultural adaptation, validation, and normalization. Journal of Epidemiology and Community Health, 57, 259-263. doi:10.1136/jech.57.4.259

[23] Ren, X.S., Amick, B., Zhou, L. and Gandek, B. (1998) Translation and psychometric evaluation of a Chinese version of the SF-36 Health Survey in the United States. Journal of Clinical Epidemiology, 51, 1129-1138. doi:10.1016/S0895-4356(98)00104-8

[24] Chen, T.H., Li, L., Sigle, J.M., Du, Y.P., Wang, H.M. and Lei, J. (2007) Crossover randomized controlled trial of the electronic version of the Chinese SF-36. Journal of Zhejiang University Science, 8, 604-608. doi:10.1631/jzus.2007.B0604

[25] Li, Y., Li, W.T., Fan, B.H. and Fu, H. (2004) Physical activity prevalence study in Shanghai city. Chinese Journal of Industrial Hygiene and Occupational Diseases, 22, 458-460.

[26] Wang, R., Wu, C., Zhao, Y., Yan, X., Ma, X., Wu, M., et al. (2008) Health related quality of life measured by SF-36: A population-based study in Shanghai, China. BMC Public Health, 8, 292. doi:10.1186/1471-2458-8-292

[27] Brown, D.W., Balluz, L.S., Heath, G.W., Moriarty, D.G., Ford, E.S., Giles, W.H. and Mokdad, A.H.(2003) Associations between recommended levels of physical activity and health-related quality of life. Findings from the
2001 Behavioral Risk Factor Surveillance System (BRFSS) survey. Preventive medicine, 37, 520-528. doi:10.1016/S0091-7435(03)00179-8

[28] Morimoto, T., Oguma, Y., Yamazaki, S., Sokejima, S. Nakayama, T. and Fukuhara, S. (2006) Gender differences in effects of physical activity on quality of life and resource utilization. Quality of Life Research, 15, 537546. doi:10.1007/s11136-005-3033-2

[29] Wendel-Vos, G.C., Schuit, A.J., Tijhuis, M.A. and Kromhout, D. (2004) Leisure time physical activity and healthrelated quality of life: Cross-sectional and longitudinal associations. Quality of Life Research, 13, 667-677. doi:10.1023/B:QURE.0000021313.51397.33

[30] Wolin, K.Y., Glynn, R.J., Colditz, G.A., Lee, I.M. and Kawachi, I. (2007) Long-term physical activity patterns and health-related quality of life in U.S. women. American Journal of Preventive Medicine, 32, 490-499. doi:10.1016/j.amepre.2007.02.014

[31] Macera, C.A., Ham, S.A., Yore, M.M, Jones, D.A., Ainsworth, B.E., Kimsey, C.D., et al. (2005) Prevalence of physical activity in the United States: Behavioral risk factor surveillance system, 2001. Preventing Chronic Disease, 2, 1-10.

[32] Karim, A.-O. and Alfred, R. (2008) Relation of leisure time, occupational, domestic and commuting physical activity to health indicators in Europe. Preventive Medicine, 47, 319-323. doi:10.1016/j.ypmed.2008.03.012

[33] Rhodes, S.D., Bowie, D.A. and Hergenrather, K.C. (2003) Collecting behavioral data using the world wide web: considerations for researchers. Journal of Epidemiology \& Community Health, 57, 68-73. doi:10.1136/jech.57.1.68

[34] Macfarlane, D.J., Lee, C.C., Ho, E.Y., Chan, K.L. and Chan, D. (2006) Convergent validity of six methods to assess physical activity in daily life. The Journal of applied psychology, 101, 1328-1334. 